\title{
Temporal evolution of programmed death-ligand 1 expression in patients with non-small cell lung cancer
}

Chang Hyun Nam ${ }^{1}$, Jaemoon $\mathrm{Koh}^{2}$, Chan-Young Ock ${ }^{1}$, Miso Kim ${ }^{1}$, Bhumsuk Keam ${ }^{1,3}$, Tae Min Kim³, Yoon Kyung Jeon ${ }^{2}$, Dong-Wan Kim ${ }^{1,3}$, Doo Hyun Chung ${ }^{2}$, and Dae Seog Heo ${ }^{1,3}$

Departments of ${ }^{1}$ Internal Medicine and ${ }^{2}$ Pathology, Seoul National University Hospital, Seoul; ${ }^{3}$ Cancer Research Institute, Seoul National University College of Medicine, Seoul, Korea

Received: April 25, 2020

Revised : July 8, 2020

Accepted: July 12, 2020

\section{Correspondence to} Chan-Young Ock, M.D.

Department of Internal

Medicine, Seoul National

University Hospital, 101

Daehak-ro, Jongno-gu, Seoul

03080, Korea

Tel: +82-2-2072-7367

Fax: +82-2-762-9662

E-mail: ock.chanyoung@gmail.com https://orcid.org/0000-0002-

6755-9728
Background/Aims: Programmed death-ligand 1 (PD-L1) expression, a validated predictive biomarker for anti-PD-1/PD-L1 inhibitors, is reported to change over time. This poses challenges during clinical application in non-small cell lung cancer.

Methods: This study included patients with non-small cell lung cancer who underwent surgery or biopsy and evaluation of PD-Lı expression in tumor cells via immunohistochemistry more than twice. We set the threshold of PD-L1 positivity to $10 \%$ and categorized patients into four groups according to changes in PD-L1 expression. Clinicopathologic information was collected from medical records. Statistical analyses, including Fisher's exact test and log-rank test, were performed.

Results: Of 109 patients, 38 (34.9\%) and 45 (41.3\%) had PD-L1 positivity in archival and recent samples, respectively. PD-L1 status was maintained in 78 (71.6\%) patients, but changed in $31(28.4 \%)$, with 19 (17.4\%) from negative to positive. There were no significant differences in characteristics between patients who maintained PD-Lı negativity and whose PD-Lı status changed from negative to positive. Patients harboring PD-L1 positivity in either archival or recent samples achieved better responses $(p=0.129)$ and showed longer overall survival than those who maintained PD-L1 negativity when they received immune checkpoint inhibitors after platinum failure (median overall survival 14.4 months vs. 4.93 months; hazard ratio, $0.43 ; 95 \%$ confidence interval, 0.20 to 0.93 ).

Conclusions: PD-L1 status changed in about one-fourth of patients. PD-L1 positivity in either archival or recent samples was predictive of better responses to immune checkpoint inhibitors. Therefore, archival samples could be used for assessment of PD-L1 status. The need for new biopsies should be decided individually.

Keywords: B7-H1 antigen; Immunotherapy; Non-small cell lung cancer

\section{INTRODUCTION}

Cancer cells evade the immune system by multiple mechanisms, one of which involves immune-inhibitory pathways, called immune checkpoints, which normally function in the maintenance of immune homeostasis [1]. 
Blockade of immune checkpoints for augmenting antitumor immunity has emerged as a promising approach to novel cancer treatment. Clinical trials using immune checkpoint inhibitors demonstrated durable objective responses and extended overall survival (OS) compared to cytotoxic chemotherapies in patients with non-small cell lung cancer (NSCLC). This supported the approval of anti-programmed death-ligand 1 (anti-PD-L1)/PD-L1 drugs by the U.S. Food and Drug Administration [2,3].

However, immune checkpoint inhibitors are not effective in all patients, highlighting the investigation of predictive biomarkers for the optimization of therapeutic strategies [4]. To date, various biomarker signatures have been developed, such as PD-L1 expression [5], tumor-infiltrating lymphocytes [6], mutational burden [7], immune cytolytic activity score [8], interferon- $\gamma$ signature [9], and immune signature [10].

Assessing tumor cell PD-L1 expression for the prediction of response to anti-PD-1/PD-L1 therapy seems rational; moreover, clinical trials have demonstrated a significant association between the level of PD-Li expression and efficacy of anti-PD-1 drugs in NSCLC $[2,3]$. However, several limitations on the use of PD-L1 expression as a predictive biomarker were observed, including temporal changes, which indicated that PDL1 expression appeared to change over time as cancer progressed and as anti-cancer treatments were administered $[11,12]$. This raises the question of whether newly acquired samples are mandatory for the accurate determination of PD-L1 status, or it is still clinically beneficial to determine the PD-L1 status using previously collected samples. To address this problem, a previous study compared the PD-Li status between archival and recent samples in patients with NSCLC, and reported a concordance rate of $67 \%$; however, they did not evaluate how changes in PD-Li expression affected the response to immune checkpoint inhibitors [11]. Another study compared the predictive value of $\mathrm{PD}-\mathrm{L} 1$ expression in archival and recent samples, and showed that PD-L1 status was predictive of the clinical benefits regardless of the age of the sample [13]. In this study, we analyzed the clinical characteristics of patients with NSCLC, who underwent surgery or biopsy for tumor samples, and evaluated the PD-Li expression status by immunohistochemistry (IHC) assays more than twice. We assessed the concordance rate of PD-L1 status, clinicopathologic characteristics related to changes in PD-L1 expression, and differences in response to immune checkpoint inhibitors according to the temporal changes in $\mathrm{PD}-\mathrm{L} 1$ expression.

\section{METHODS}

\section{Study population}

Among the patients with NSCLC who visited Seoul National University Hospital from 2010 to 2018, those who underwent surgery or biopsy for tumor samples, and evaluation of $\mathrm{PD}-\mathrm{L} 1$ expression via IHC assays more than twice were selected based on the data retrieved from the Seoul National University Hospital Patients Research Environment system. For the IHC assay, any of the following antibodies, $22 \mathrm{C}_{3}$ (Dako, Carpinteria, CA, USA), 28-8 (Dako), E1L3N (Cell Signaling Technology, Danvers, MA, USA), SP142 (Ventana, Tucson, AZ, USA), or $\mathrm{SP}_{2} 6_{3}$ (Ventana) could be used for the assessment of PD-L1 expression. In addition, for patients who were administered immune checkpoint inhibitors and evaluated for PD-Li expression using recently collected samples, the PD-Li expression analysis was performed on archival samples only if they were available.

We retrospectively analyzed the clinicopathologic information from the electronic medical records. PD-L1 positivity was determined using a threshold of 10\% for PD-L1 expression in tumor cells, called Tumor Proportion Score (TPS) [3], which is one of the conservative cutoff values suggested in a previous study [2]. The patients were divided into four groups according to the PD-L1 expression status in archival and recent samples: the negative to negative (PD-L1 [-/-] group), negative to positive (PD-L1 [-/+] group), positive to negative (PD-L1 [+/-] group), and positive to positive (PD-L1 $[+/+]$ group). Responses to immune checkpoint inhibitors were assessed using the Response Evaluation Criteria In Solid Tumors version 1.1 [14].

\section{Statistical analysis}

The patient characteristics were compared using the chi-square test or Fisher's exact test, as appropriate. Survival analyses were performed using the Kaplan-Meier method, log-rank test, and Cox proportional hazards model, and summarized as the hazard ratio (HR) and 
95\% confidence interval (CI). All tests were two-tailed and a $p<0.05$ was considered statistically significant. All statistical analyses were performed using SPSS Statistics for Windows version 25.0 (IBM Corp., Armonk, NY, USA).

\section{Ethical statement}

This study was approved by the Institutional Review Board at Seoul National University Hospital (changes in PD-L1 expression after chemotherapy in patients with non-small cell lung cancer, IRB no. H-1902-093-1010). Written informed consent from patients was waived as this was a retrospective study.

\section{RESULTS}

\section{Patient characteristics}

A total of 99 patients with NSCLC underwent surgery or biopsy, and evaluation of PD-L1 expression more than twice. In addition, 10 patients who underwent additional PD-L1 tests on archival samples using the $\mathrm{SP}_{2} 63$ assay were also analyzed. Overall, about $70 \%$ of the samples were evaluated using the $\mathrm{SP}_{2} 6_{3}$ assay (Supplementary Table 1), and the concordance of IHC assays used in archival and recent samples was $56.9 \%$. The baseline characteristics of the patients are summarized in Table 1. Overall, the median age of patients was 67 years (range, 29 to 88 ), and 77 (70.6\%) were males. More than half of the patients $(64.2 \%)$ had adenocarcinoma, and the majority of patients were initially diagnosed with stage III (19.3\%) or IV (62.4\%) lung cancer. The median follow-up duration was 32.1 months (range, 2.3 to 162.2). Six patient

Table 1. Patient characteristics

\begin{tabular}{|c|c|c|c|c|c|c|}
\hline Characteristic & $\begin{array}{c}\mathrm{PD}-\mathrm{L1}(-/-) \\
(\mathrm{n}=52)\end{array}$ & $\begin{array}{c}\mathrm{PD}-\mathrm{L1}(-/+) \\
(\mathrm{n}=19)\end{array}$ & $\begin{array}{c}\mathrm{PD}-\mathrm{L1}(+/-) \\
(\mathrm{n}=12)\end{array}$ & $\begin{array}{c}\mathrm{PD}-\mathrm{L} 1(+/+) \\
(\mathrm{n}=26)\end{array}$ & $\begin{array}{c}\text { Total } \\
(\mathrm{n}=109)\end{array}$ & $p$ value \\
\hline Age, yr & $65.5(29-88)$ & $69(53-80)$ & $73(44-88)$ & $64(49-81)$ & $67(29-88)$ & 0.271 \\
\hline Male sex & $33(63.5)$ & $14(73.7)$ & $10(83 \cdot 3)$ & $20(76.9)$ & $77(70.6)$ & 0.477 \\
\hline Smoking status & & & & & & 0.727 \\
\hline Current/former smokers & $32(61.5)$ & $13(68.4)$ & $9(75.0)$ & $19(73.1)$ & $73(67.0)$ & \\
\hline Never smokers & $20(38.5)$ & $6(31.6)$ & $3(25.0)$ & $7(26.9)$ & $36(33.0)$ & \\
\hline ECOG at diagnosis & & & & & & 0.412 \\
\hline o & $17(32.7)$ & $9(47 \cdot 4)$ & $1(8.3)$ & $10(38.5)$ & $37(34.0)$ & \\
\hline 1 & $31(59.6)$ & $9(47.4)$ & $10(83 \cdot 3)$ & $14(53.8)$ & $64(58.7)$ & \\
\hline 2 & $4(7 \cdot 7)$ & $1(5 \cdot 3)$ & $1(8.3)$ & $2(7 \cdot 7)$ & $8(7 \cdot 3)$ & \\
\hline Histology & & & & & & 0.085 \\
\hline Adenocarcinoma & $34(65.4)$ & $12(63.2)$ & $4(33 \cdot 3)$ & $20(76.9)$ & $70(64.2)$ & \\
\hline Others & $18(34.6)$ & $7(36.8)$ & $8(66.7)$ & $6(23.1)$ & $39(35.8)$ & \\
\hline Disease stage at diagnosis & & & & & & 0.237 \\
\hline I & $5(9.6)$ & $3(15.8)$ & $2(16.7)$ & $2(7 \cdot 7)$ & $12(11.0)$ & \\
\hline II & $4(7 \cdot 7)$ & $1(5 \cdot 3)$ & $3(25 \cdot 0)$ & o & $8(7 \cdot 3)$ & \\
\hline III & $9(17 \cdot 3)$ & $6(31.6)$ & $1(8.3)$ & $5(19.2)$ & $21(19 \cdot 3)$ & \\
\hline IV & $34(65 \cdot 4)$ & $9(47 \cdot 4)$ & $6(50.0)$ & $19(73.1)$ & $68(62.4)$ & \\
\hline \multicolumn{7}{|l|}{ Status of oncogenic alteration } \\
\hline EGFR mutation & $17(32.7)$ & $7(36.8)$ & $2(16.7)$ & $5(19.2)$ & $31(28.4)$ & 0.432 \\
\hline Others & $4(7 \cdot 7)$ & $1(5 \cdot 3)$ & $1(8.3)$ & $6(23.1)$ & $12(11.0)$ & 0.183 \\
\hline Follow-up duration, mo & $25.53(2.3-111.2)$ & $43.0(9.3-162.2)$ & $38.1(19.2-86.2)$ & $23.8(8.5-49.1)$ & $32.1(2.3-162.2)$ & 0.140 \\
\hline
\end{tabular}

Values are presented as median (range) or number (\%).

PD-L1, programmed death-ligand 1; ECOG, Eastern Cooperative Oncology Group; EGFR, epidermal growth factor receptor. 
Table 2. Comparison of clinicopathologic characteristics between patients in the PD-L1 (-/-) and PD-L1(-/+) groups

\begin{tabular}{|c|c|c|c|}
\hline Characteristic & $\mathrm{PD}-\mathrm{L} 1(-/-)(\mathrm{n}=52)$ & $\mathrm{PD}-\mathrm{L} 1(-/+)(\mathrm{n}=19)$ & $p$ value \\
\hline Presence of oncogenic alterations & $21(40.4)$ & $8(42.1)$ & 0.525 \\
\hline Interval between samplings, mo & $13.7(1.2-69 \cdot 3)$ & $18.0(3.4-46.7)$ & 0.264 \\
\hline No. of chemotherapy regimens during the interval & & & 0.603 \\
\hline o & $4(7 \cdot 7)$ & $2(10.5)$ & \\
\hline 1 & $25(48.1)$ & $11(57 \cdot 9)$ & \\
\hline 2 or more & $23(44.2)$ & $6(31.6)$ & \\
\hline \multicolumn{4}{|l|}{ Type of chemotherapeutic drugs during the interval } \\
\hline Platinum & $36(69.2)$ & $12(63.2)$ & 0.775 \\
\hline Taxane & $15(28.8)$ & $7(36.8)$ & 0.569 \\
\hline EGFR TKI & $11(21.2)$ & $4(21.1)$ & 1.000 \\
\hline ALK TKI & o & $1(5 \cdot 3)$ & 0.268 \\
\hline Others & $40(76.9)$ & $12(63.2)$ & 0.364 \\
\hline No. of patients received radiotherapy during the interval & $20(38.5)$ & $8(42.1)$ & 0.342 \\
\hline
\end{tabular}

Values are presented as number (\%) or median (range).

PD-L1, programmed death-ligand 1; EGFR, epidermal growth factor receptor; TKI, tyrosine kinase inhibitor; ALK, anaplastic lymphoma kinase.

samples were assessed for PD-L1 expression at least three times; for such patient samples, the earliest and latest ones were included in the analysis.

Among 109 patients, 52 (47.7\%) maintained negative PD-L1 status in both archival and recent samples (PD-L1 [-/-] group), 19 (17.4\%) showed changes in PDL1 status from negative in the archival sample to positive in the recent sample (PD-L1 [-/+] group), 12 (11.0\%) showed changes in PD-L1 status from positive in the archival sample to negative in the recent sample (PDL1 [+/-] group), and 26 (23.9\%) retained PD-L1 positivity in both archival and recent samples (PD-L1 [+/+] group). In other words, 38 (34.9\%) patients in archival samples and $45(41.3 \%)$ in recent samples showed PD-L1 positivity. In addition, 78 (71.6\%) patients maintained their PDL1 status, whereas 31 (28.4\%) showed changes in PD-L1 status, with 19 (17.4\%) showing changes in PD-L1 status from negative to positive. When the patients in the four groups were compared, no significant differences in major clinicopathologic characteristics were observed (Table 1, Supplementary Fig. 1).

\section{Comparison of characteristics of patients with PD- Lı (-/-) versus PD-Lı (-/+) statuses}

To assess the clinicopathologic characteristics relevant to the positive conversion of $\mathrm{PD}-\mathrm{L} 1$, we compared patients in the PD-L1 (-/-) group with those in the PD$\mathrm{L} 1(-/+)$ group. Patients in the PD-L1 (-/-) group mostly received taxane-based chemotherapy and radiotherapy between the two biopsy time points; however, there were no significant differences in characteristics between the two groups (Table 2). Brief clinical information of patients with positive conversion of PD-L1 is described in Supplementary Table 2.

\section{Responses to immune checkpoint inhibitors}

Among 109 patients included in this study, 56 were given immune checkpoint inhibitors after failure of platinum-based therapy. The number of patients who belonged to the PD-L1 (-/-), PD-Li (-/+), PD-Li (+/-), and $\mathrm{PD}-\mathrm{L} 1(+/+)$ groups were $18,11,7$, and 20 patients, respectively. Forty-one (73.2\%) patients were administered nivolumab, while eight (14.3\%) and seven (12.5\%) patients were administered pembrolizumab and atezolizumab, respectively. There were no significant differences in the pre-treatment characteristics such as the Eastern Cooperative Oncology Group (ECOG) performance status, number of prior chemotherapy regimens, type of prior chemotherapy, and follow-up durations among patients in the four groups (Table 3). 
Table 3. Pre-treatment characteristics and best responses to immune checkpoint inhibitors

\begin{tabular}{|c|c|c|c|c|c|}
\hline Characteristic & $\begin{array}{l}\mathrm{PD}-\mathrm{Lr}(-/-) \\
\quad(\mathrm{n}=18)\end{array}$ & $\begin{array}{l}\mathrm{PD}-\mathrm{L} 1(-/+) \\
\quad(\mathrm{n}=11)\end{array}$ & $\begin{array}{l}\mathrm{PD}-\mathrm{L} 1(+/-) \\
\quad(\mathrm{n}=7)\end{array}$ & $\begin{array}{l}\mathrm{PD}-\mathrm{L}_{1}(+/+) \\
(\mathrm{n}=20)\end{array}$ & $p$ value \\
\hline ECOG & & & & & 0.275 \\
\hline 0 & 0 & $2(18.2)$ & $1(14 \cdot 3)$ & $2(10.0)$ & \\
\hline 1 or more & $18(100)$ & $9(81.8)$ & $6(85 \cdot 7)$ & $18(90.0)$ & \\
\hline No. of prior chemotherapy regimens & & & & & 0.572 \\
\hline 1 & $6(33 \cdot 3)$ & $5(45 \cdot 5)$ & $3(42.9)$ & $9(45 \cdot 0)$ & \\
\hline 2 & $6(33 \cdot 3)$ & $2(18.2)$ & $4(57 \cdot 1)$ & $4(20.0)$ & \\
\hline 3 & $3(16.7)$ & $2(18.2)$ & o & $6(30.0)$ & \\
\hline 4 or more & $3(16.7)$ & $2(18.2)$ & o & $1(5.0)$ & \\
\hline \multicolumn{6}{|l|}{ Type of prior chemotherapy } \\
\hline Platinum-based therapy & $18(100)$ & $11(100)$ & $7(100)$ & $20(100)$ & NA \\
\hline EGFR-TKI & $3(16.7)$ & $4(36.4)$ & 0 & $4(20.0)$ & 0.288 \\
\hline ALK-TKI & 0 & 0 & 0 & $2(10.0)$ & 0.495 \\
\hline $\begin{array}{l}\text { Interval between recent sampling and } \\
\text { ICI start, mo }\end{array}$ & $0.9(0-13.3)$ & $3.5(0-11.9)$ & $0.4(0.1-13.7)$ & $3.2(0.1-11.4)$ & 0.363 \\
\hline Follow-up duration, mo & $4.9(0.4-14.8)$ & $7.9(0.7-30.6)$ & $14.5(3.7-26.8)$ & $8.3(0.7-30.9)$ & 0.093 \\
\hline Objective response rate & 0 & $2(18.2)$ & $1(14 \cdot 3)$ & $6(30.0)$ & 0.054 \\
\hline Best response & & & & & 0.129 \\
\hline Complete response & o & 0 & $\mathrm{O}$ & 0 & \\
\hline Partial response & 0 & $2(18.2)$ & $1(14 \cdot 3)$ & $6(30.0)$ & \\
\hline Stable disease & $7(38.9)$ & $4(36.4)$ & $3(42.9)$ & $2(10.0)$ & \\
\hline Progressive disease & $8(44 \cdot 4)$ & $4(36.4)$ & $3(42.9)$ & $11(55 \cdot 0)$ & \\
\hline Unable to determine & $3(16.7)$ & $1(9.1)$ & 0 & $1(5.0)$ & \\
\hline
\end{tabular}

Values are presented as number (\%) or median (range).

PD-L1, programmed death-ligand 1; ECOG, Eastern Cooperative Oncology Group; NA, not applicable; EGFR, epidermal growth factor receptor; TKI, tyrosine kinase inhibitor; ALK, anaplastic lymphoma kinase; ICI, immune checkpoint inhibitor.

Notably, only patients in the PD-Li (-/+), PD-Li (+/-), and PD-L1 (+/+) groups had an objective response to immune checkpoint inhibitors, and showed objective response rates of $18.2 \%, 14.3 \%$, and $30.0 \%$, respectively. None of the patients in the PD-L1 (-/-) group achieved an objective response (o\%). In addition, patients with PD-L1 positivity in any of the archival or recent samples (PD-L1 [+, any] group) showed a median OS of 14.4 months, which was significantly longer than that of those who maintained negative PD-L1 status in both archival and recent samples (PD-L1 [-/-] group) (median OS, 4.93 months; HR, 0.43 ; 95\% CI, 0.20 to 0.93 ). According to multivariate analysis, $\mathrm{PD}-\mathrm{L} 1$ positivity in any samples showed risk reduction in OS (HR, 0.31; 95\%
CI, 0.10 to $0.90 ; p=0.031$ ), regardless of other factors including age, sex, smoking status, histological subtype, EGFR mutation, number of prior chemotherapy regimens, and pre-treatment ECOG (Supplementary Table 3). There were no significant differences in progression-free survival (PFS) between the two groups (2.13 months vs. 1.07 months in the PD-L1 [+, any] and PD-L1 [-/-] groups, respectively; HR, 0.55; 95\% CI, 0.30 to 1.03) (Fig. 1). When each of the four groups were compared separately, patients in the PD-L1 (-/+), PD-L1 (+/-), and PD-L1 (+/+) groups tended to have longer OS and PFS, but the differences were not statistically significant (Fig. 2). Using multivariate analysis, patients in the PD-L1 (-/+), PD-L1 (+/-), and PD-L1 (+/+) groups showed similar 


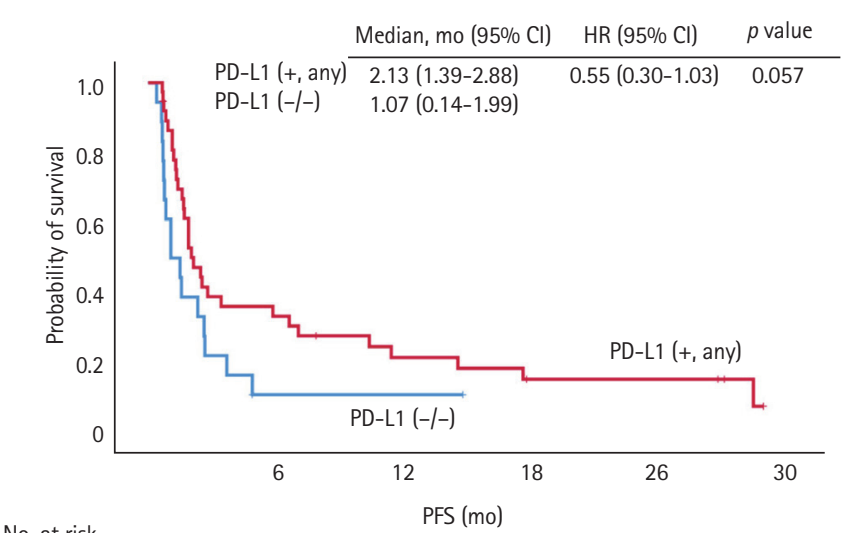

No. at risk

PD-L1 (+, any) 38 PD-L1 (-l-) 18

A

Figure 1. Comparison of responses to immune checkpoint inhibitors between patients with programmed death-ligand 1 (PD$\mathrm{L1})$ positivity in either archival or recent samples (PD-L1 [+, any] group) and those with negative PD-L1 status in both samples (PD-L1 [-/-] group). (A) The progression-free survival (PFS) was longer in the PD-L1 (+, any) group than in the PD-L1 (-/-) group (median PFS, 2.13 months vs. 1.07 months; hazard ratio [HR], 0.55; 95\% confidence interval [CI], 0.30 to 1.03), but without statistical significance. (B) The overall survival (OS) was significantly longer in the PD-L1 (+, any) group than in the PD-L1 (-/-) group (median OS, 14.4 months vs. 4.93 months; HR, 0.43; 95\% CI, 0.20 to 0.93).

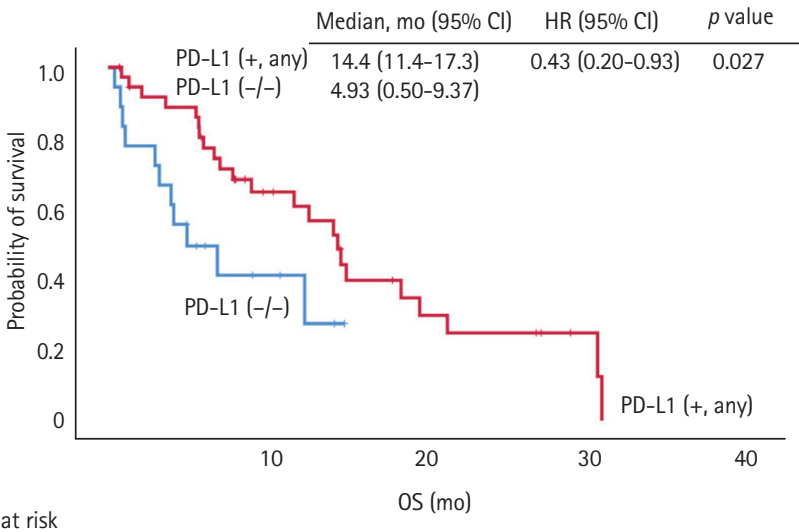

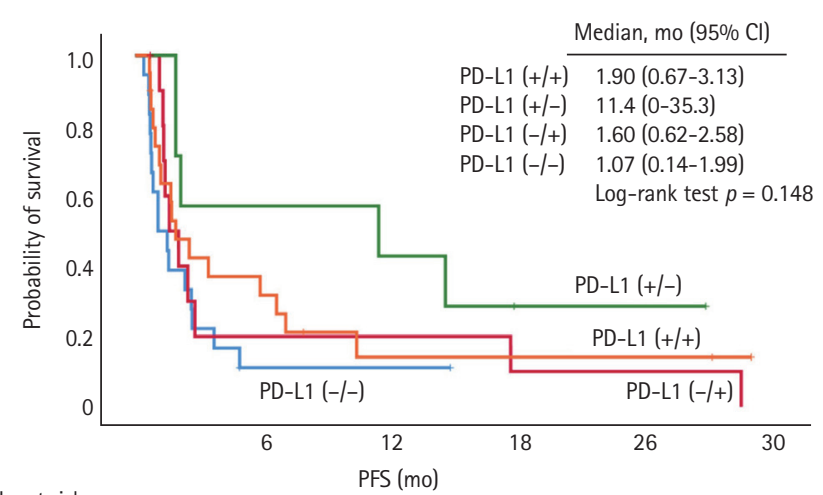

\begin{tabular}{crrcccc} 
No. at risk & \multicolumn{7}{c}{ PFS (mo) } \\
PD-L1(+/+) & 20 & 6 & 2 & 2 & 2 & 0 \\
PD-L1 (+/-) & 7 & 4 & 3 & 1 & 1 & 0 \\
PD-L1 (-/+) & 11 & 2 & 2 & 1 & 1 & 0 \\
PD-L1 (-/-) & 18 & 1 & 1 & 0 & 0 & 0
\end{tabular}

A

Figure 2. Comparison of responses to immune checkpoint inhibitors among patients in the four groups categorized according to the changes in programmed death-ligand 1 (PD-L1) expression. There were no significant differences in (A) progression-free survival (PFS) and (B) overall survival (OS) among patients in the four groups. CI, confidence interval.

amounts of risk reduction in OS, but they were not statistically significant (Supplementary Table 4).

In a comparison of patients according to PD-L1 positivity in the archival sample (pre-PD-L1 [+] group vs. pre-

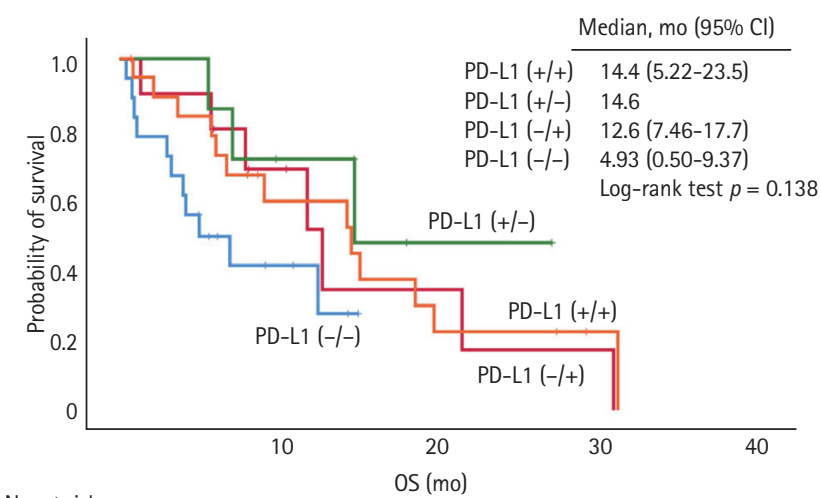

No. at risk

PD-L1(+/+) 20

PD-L1 (+/-) 7

PD-L1 (-/+) 11

PD-L1 (-/-) 18

$\begin{array}{llll}8 & 3 & 1 & 0 \\ 4 & 1 & 0 & 0 \\ 5 & 2 & 1 & 0 \\ 4 & 0 & 0 & 0\end{array}$

\section{B}


months [HR, 0.59; 95\% CI, 0.33 to 1.06]) (Supplementary Fig. 2). There were no significant differences in the comparison of patients by $\mathrm{PD}-\mathrm{L} 1$ positivity in the recent sample (post-PD-L1 [+] group vs. post-PD-L1 [-] group; median OS 14.1 months vs. 7.00 months [HR, 0.75; 95\% CI, 0.37 to 1.53], median PFS 1.90 months vs. 1.90 months [HR, 0.94; 95\% CI, 0.53 to 1.69]) (Supplementary Fig. 3).

\section{DISCUSSION}

In this study, when PD-L1 expression in archival and recent samples were compared, we found that $71.6 \%$ of patients with NSCLC maintained their PD-L1 status, whereas $28.4 \%$ showed changes in PD-L1 status, with $17.4 \%$ changing from PD-L1-negative to positive. In addition, patients showing PD-L1 positivity in any of the archival or recent samples had longer OS than those without PD-L1 positivity when they were given immune checkpoint inhibitors after failure of platinum-based therapy.

About one-fourth of patients showed changes in PDL1 status over time, which could be attributed to several factors including actual changes in PD-Li expression, intertumoral and intratumoral heterogeneity, and analytic variations generated from the use of different types of PD-L1 IHC assays. First, PD-L1 expression in tumor cells could actually change over time by innate and extrinsic mechanisms [15]. Previous studies have reported that PD-Li expression could be up-regulated by EGFR mutation [16], EML4-ALK gene fusion [17], and other genetic alterations [18], and could change after anti-cancer treatment including chemotherapy $[12,19,20]$, EGFR tyrosine kinase inhibitors [21], and radiotherapy [22] via the MAPK/ERK pathway $[17,19]$ or JAK/STAT pathway [16]. Unfortunately, we found no significant differences in clinicopathologic characteristics between the PD-L1 $(-/-)$ and PD-Li $(-/+)$ groups, although patients in the PD-L1 (-/+) group were more likely to have received taxane-based chemotherapy and radiotherapy between the two biopsy time points. Thus, further investigations are needed to elucidate the effect of chemotherapy on PDL1 expression and its underlying mechanisms.

In addition, intertumoral and intratumoral heterogeneity could contribute to the observed differences in PD-L1 expression in paired samples. Several studies compared PD-L1 expression in whole surgical tissues and matched lung biopsies and reported discordance rates of PD-L1 positivity in the range of $14 \%$ to $43 \%$ [2325 . Other studies showed that the extent of PD-L1 expression varied among tumor sites in more than negligible number of cases $[26,27]$. Such discrepancies from spatial heterogeneity possibly lead to misclassification of PD-Li status and misguide to or against the use of anti-PD-1/PD-L1 inhibitors. In this study, the biopsy sites of paired samples were not matched because it is practically difficult to eliminate errors from intratumoral heterogeneity even if we only analyze archival and recent samples from the same sites. In addition, we believe that it is closer to actual clinical situations because determining target tissue of additional biopsy is usually dependent on the accessibility to the sites or the risk of the biopsy procedure rather than keeping the previous sites.

Moreover, the observed levels of PD-Li expression are arguably dependent on the methods used to assess PD-Li expression. Several kinds of IHC assays for assessing PD-L1 expression are now available, including 22 3 3, 28-8, SP142, and SP263. The Blueprint PD-L1 IHC Assay Comparison Project showed that $22 \mathrm{C}_{3}, 28-8$, and $\mathrm{SP}_{2} 6_{3}$ had similar analytic performances, although interchanging the assays and cut-off values could result in misclassification of PD-L1 status in some patients [28]. Subsequent cross-validation studies showed consistent results [29,30]; however, no gold standard assay has yet been established, which could accurately evaluate PD$\mathrm{L} 1$ expression and predict the benefit of anti-PD-1/ PD-L1 agents [13]. In addition, the threshold of PD-L1 positivity is a controversial issue because various criteria were used in clinical trials so far and none of them were recognized as a standard. Accordingly, we decided to choose one of the PD-Li cut-off values from previous studies and set the PD-L1 positivity to $10 \%$, derived from the pivotal study of nivolumab for nonsquamous lung cancer, which is supported by the observation that most of the patients (73.2\%) who received immune checkpoint inhibitors eventually received nivolumab. Furthermore, analysis of the use of other PD-L1 cut-off values ( $1 \%$ or $20 \%$ ) showed similar results with the PD-L1 cut-off value of $10 \%$.

Despite limitations derived from temporal changes, spatial heterogeneity, and analytic variations, PD-L1 ex- 
pression measured by IHC assays is the only clinically available and validated predictive biomarker of immune checkpoint inhibition to date. Accordingly, researchers have attempted to characterize the drawbacks of PD-L1 expression and address the problems that are currently being faced, one of which is the utility of archival samples in determining PD-L1 status instead of newly collected samples. One study addressed this issue and reported a good correlation of PD-L1 expression between archival and newly collected samples [11]. In addition, the updated analysis of the KEYNOTE-o1o trial showed an OS benefit of pembrolizumab over docetaxel for both TPS $\geq 50 \%$ and $\geq 1 \%$, regardless of whether archival or newly collected samples were used for the assessment of PD-L1 status [13]. Here, we found a relatively high degree of concordance (76.6\%), and also demonstrated the predictive power of PD-L1 positivity in either archival or recent samples. These findings are consistent with previous studies and imply that archival samples could be used to determine PD-L1 status. This study has significance in that we assessed both the concordance rate of PD-L1 status and its predictive value independently of the age of samples in one group of patients.

This study has several limitations. We only assessed patients with paired biopsy samples, and this may not be representative of all the patients with NSCLC, considering that patients with negative PD-L1 status or non-responsiveness to prior chemotherapy are more likely to undergo additional biopsy with the evaluation of PD-L1 status. Moreover, it was difficult to observe statistically significant differences by changes in PD-L1 status with a relatively small number of patients. In addition, we utilized various available antibodies for IHC assays for assessing PD-Li expression and set the threshold of PD-L1 positivity to $10 \%$, which could result in misclassification of PD-L1 status in some patients. Future prospective studies with a larger number of patients using standardized assays for PD-Li expression and valid cutoff values of PD-L1 positivity will help us better understand the predictive value of $\mathrm{PD}-\mathrm{L} 1$ expression and the effect of temporal changes.

In conclusion, PD-L1 status changed in about onefourth of patients when archival and recent tumor samples were compared; and PD-L1 positivity in either archival or recent samples was predictive of better responses to immune checkpoint inhibitors. Therefore, archival samples could be used to assess PD-L1 status, and new biopsies should be considered in selected patients who are likely to benefit from additional biopsy, particularly those with negative PD-L1 status in archival samples.

\section{KEY MESSAGE}

1. Programmed death-ligand 1 (PD-L1) status changed in about one-fourth of patients when comparing archival and recent tumor samples.

2. PD-L1 positivity in either archival or recent samples was predictive of better responses to immune checkpoint inhibitors.

3. Archival samples could be used to assess PDL1 status and whether to obtain new biopsies should be decided on a patient-by-patient basis.

\section{Conflict of interest}

No potential conflict of interest relevant to this article was reported.

\section{Acknowledgments}

This study was supported by a grant of the Korea Health Technology R\&D Project "Strategic Center of Cell and Bio Therapy for Heart, Diabetes \& Cancer" through the Korea Health Industry Development Institute (KHIDI), funded by the Ministry of Health \& Welfare (MHW), Republic of Korea (grant number: $\mathrm{HI} 17 \mathrm{C} 2085$ ).

\section{REFERENCES}

1. Pardoll DM. The blockade of immune checkpoints in cancer immunotherapy. Nat Rev Cancer 2012;12:252-264.

2. Borghaei H, Paz-Ares L, Horn L, et al. Nivolumab versus docetaxel in advanced nonsquamous non-small-cell lung cancer. N Engl J Med 2015;373:1627-1639.

3. Herbst RS, Baas P, Kim DW, et al. Pembrolizumab versus docetaxel for previously treated, PD-L1-positive, advanced non-small-cell lung cancer (KEYNOTE-O10): a randomised controlled trial. Lancet 2016;387:1540-1550.

4. Gibney GT, Weiner LM, Atkins MB. Predictive biomarkers for checkpoint inhibitor-based immunotherapy. Lancet Oncol 2016;17:e542-e551. 
5. Topalian SL, Hodi FS, Brahmer JR, et al. Safety, activity, and immune correlates of anti-PD-1 antibody in cancer. N Engl J Med 2012;366:2443-2454.

6. Ock CY, Keam B, Kim S, et al. Pan-cancer immunogenomic perspective on the tumor microenvironment based on PD-L1 and CD8 T-cell infiltration. Clin Cancer Res 2016;22:2261-2270.

7. Rizvi NA, Hellmann MD, Snyder A, et al. Cancer immunology. Mutational landscape determines sensitivity to PD-1 blockade in non-small cell lung cancer. Science 2015;348:124-128.

8. Rooney MS, Shukla SA, Wu CJ, Getz G, Hacohen N. Molecular and genetic properties of tumors associated with local immune cytolytic activity. Cell 2015;160:48-61.

9. Ayers M, Lunceford J, Nebozhyn M, et al. IFN- $\gamma$-related mRNA profile predicts clinical response to PD-1 blockade. J Clin Invest 2017;127:2930-2940.

10. Ock CY, Hwang JE, Keam B, et al. Genomic landscape associated with potential response to anti-CTLA-4 treatment in cancers. Nat Commun 2017;8:1050.

11. Cho JH, Sorensen SF, Choi YL, et al. Programmed death ligand 1 expression in paired non-small cell lung cancer tumor samples. Clin Lung Cancer 2017;18:e473-e479.

12. Sheng J, Fang W, Yu J, et al. Expression of programmed death ligand-1 on tumor cells varies pre and post chemotherapy in non-small cell lung cancer. Sci Rep 2016;6:20090.

13. Herbst RS, Baas P, Perez-Gracia JL, et al. Use of archival versus newly collected tumor samples for assessing PDL1 expression and overall survival: an updated analysis of KEYNOTE-o1o trial. Ann Oncol 2019;30:281-289.

14. Eisenhauer EA, Therasse P, Bogaerts J, et al. New response evaluation criteria in solid tumours: revised RECIST guideline (version 1.1). Eur J Cancer 2009;45:228-247.

15. Topalian SL, Taube JM, Anders RA, Pardoll DM. Mechanism-driven biomarkers to guide immune checkpoint blockade in cancer therapy. Nat Rev Cancer 2016;16:275287.

16. Zhang N, Zeng Y, Du W, et al. The EGFR pathway is involved in the regulation of $\mathrm{PD}-\mathrm{L} 1$ expression via the IL-6/ JAK/STAT3 signaling pathway in EGFR-mutated nonsmall cell lung cancer. Int J Oncol 2016;49:1360-1368.

17. Ota K, Azuma K, Kawahara A, et al. Induction of PD-L1 expression by the EML4-ALK oncoprotein and downstream signaling pathways in non-small cell lung cancer. Clin Cancer Res 2015;21:4014-4021.
18. Scheel AH, Ansen S, Schultheis AM, et al. PD-L1 expression in non-small cell lung cancer: correlations with genetic alterations. Oncoimmunology 2016;5:e1131379.

19. Ock CY, Kim S, Keam B, et al. Changes in programmed death-ligand 1 expression during cisplatin treatment in patients with head and neck squamous cell carcinoma. Oncotarget 2017;8:97920-97927.

20. Shin J, Chung JH, Kim SH, et al. Effect of platinum-based chemotherapy on PD-L1 expression on tumor cells in non-small cell lung cancer. Cancer Res Treat 2019;51:10861097.

21. Han JJ, Kim DW, Koh J, et al. Change in PD-L1 expression after acquiring resistance to gefitinib in EGFR-mutant non-small-cell lung cancer. Clin Lung Cancer 2016;17:263270.

22. Illidge $\mathrm{T}$, Lipowska-Bhalla $\mathrm{G}$, Cheadle E, et al. Radiation therapy induces an adaptive upregulation of $\mathrm{PD}-\mathrm{L} 1$ on tumor cells which may limit the efficacy of the anti-tumor immune response but can be circumvented by anti-PD-L1. Int J Radiat Oncol Biol Phys 2014;90(1 Suppl):S776.

23. Ilie $\mathrm{M}$, Long-Mira E, Bence $\mathrm{C}$, et al. Comparative study of the PD-L1 status between surgically resected specimens and matched biopsies of NSCLC patients reveal major discordances: a potential issue for anti-PD-L1 therapeutic strategies. Ann Oncol 2016;27:147-153.

24. Bigras G, Mairs S, Swanson PE, Morel D, Lai R, Izevbaye I. Small biopsies misclassify up to $35 \%$ of PD-L1 assessments in advanced lung non-small cell lung carcinomas. Appl Immunohistochem Mol Morphol 2018;26:701-708.

25. Kim H, Kwon HJ, Park SY, Park E, Chung JH. PD-Lı immunohistochemical assays for assessment of therapeutic strategies involving immune checkpoint inhibitors in non-small cell lung cancer: a comparative study. Oncotarget 2017;8:98524-98532.

26. Zhou J, Gong Z, Jia Q, Wu Y, Yang ZZ, Zhu B. Programmed death ligand 1 expression and CD8+ tumor-infiltrating lymphocyte density differences between paired primary and brain metastatic lesions in non-small cell lung cancer. Biochem Biophys Res Commun 2018;498:751-757.

27. Kim S, Koh J, Kwon D, et al. Comparative analysis of PDL1 expression between primary and metastatic pulmonary adenocarcinomas. Eur J Cancer 2017;75:141-149.

28. Hirsch FR, McElhinny A, Stanforth D, et al. PD-L1 immunohistochemistry assays for lung cancer: results from phase 1 of the blueprint PD-L1 IHC assay comparison 
project. J Thorac Oncol 2017;12:208-222.

29. Scheel AH, Dietel M, Heukamp LC, et al. Harmonized PD-L1 immunohistochemistry for pulmonary squamous-cell and adenocarcinomas. Mod Pathol 2016;29:1165-1172.
30. Ratcliffe MJ, Sharpe A, Midha A, et al. Agreement between programmed cell death ligand-1 diagnostic assays across multiple protein expression cutoffs in non-small cell lung cancer. Clin Cancer Res 2017;23:3585-3591. 
$\mathrm{Nam} \mathrm{CH}$, et al. PD-L1 expression changes in NSCLC

Supplementary Table 1 . The usage of various kinds of IHC assays for the assessment of PD-L1 expression

\begin{tabular}{lccc}
\hline & Archival sample & Recent sample & Total \\
\hline $\mathrm{SP}_{2} 6_{3}$ & $65(59.6)$ & $88(80.7)$ & $153(70.2)$ \\
$22 \mathrm{C}_{3}$ & - & $6(5.5)$ & $6(2.8)$ \\
$\mathrm{E}_{1} \mathrm{~L}_{3} \mathrm{~N}$ & $44(40.4)$ & $14(12.8)$ & $58(26.6)$ \\
$\mathrm{SP}_{142}$ & - & $1(0.9)$ & $1(0.5)$ \\
\hline
\end{tabular}

Values are presented as number (\%).

IHC, immunohistochemistry; PD-L1, programmed deathligand 1. 


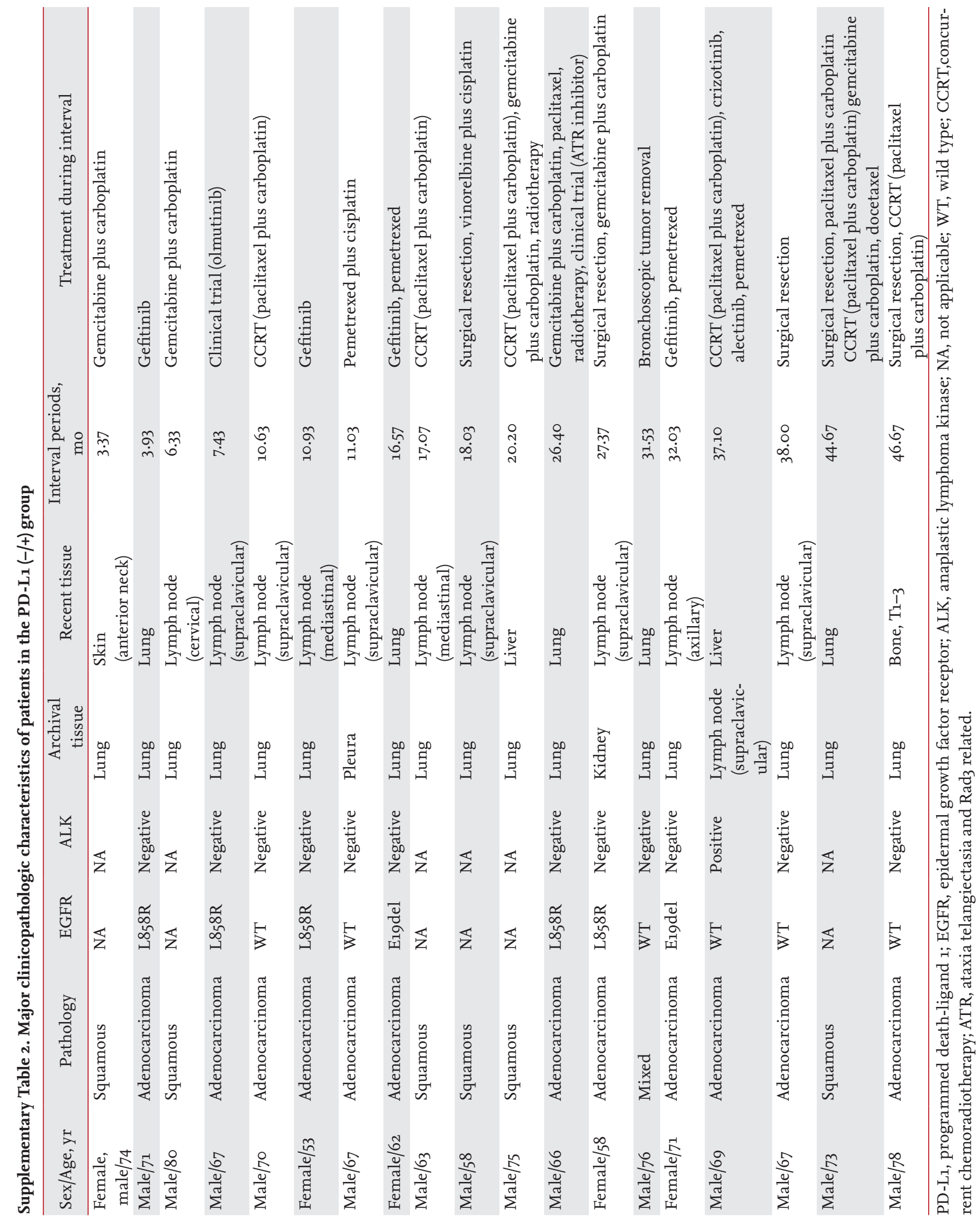


Supplementary Table 3. Multivariate analysis of overall survival after the administration of immune checkpoint inhibitor considering PD-L1 any positivity

\begin{tabular}{|c|c|c|c|c|c|c|}
\hline \multirow{2}{*}{ Parameter } & \multicolumn{3}{|c|}{ Univariate analysis } & \multicolumn{3}{|c|}{ Multivariate analysis } \\
\hline & HR & $95 \% \mathrm{CI}$ & $p$ value & HR & $95 \% \mathrm{CI}$ & $p$ value \\
\hline Age & 0.99 & $0.96-1.03$ & 0.681 & 1.04 & $0.99-1.10$ & 0.126 \\
\hline Sex (female as reference) & 0.40 & $0.19-0.82$ & $0.013^{\mathrm{a}}$ & $3 \cdot 41$ & $0.35-33.4$ & 0.291 \\
\hline \multicolumn{7}{|l|}{ Smoking status (never smoker as reference) } \\
\hline Current/former smokers & 0.40 & $0.19-0.83$ & $0.014^{\mathrm{a}}$ & 0.08 & $0.01-0.86$ & $0.037^{\mathrm{a}}$ \\
\hline Histologic subtype (adenocarcinoma as reference) & & & 0.163 & & & 0.095 \\
\hline Squamous & 0.76 & $0.34^{-1.66}$ & 0.484 & 1.47 & $0.54-4.03$ & 0.456 \\
\hline Others & 0.31 & $0.09-1.06$ & 0.061 & 0.31 & $0.08-1.13$ & 0.075 \\
\hline EGFR mutation (absent as reference) & 2.24 & $1.05-4.79$ & $0.038^{\mathrm{a}}$ & 2.37 & $0.78-7.20$ & 0.128 \\
\hline \multicolumn{7}{|l|}{ No. of prior chemotherapy regimen ( 1 or 2 as reference) } \\
\hline 3 or more & 2.22 & $1.09-4.53$ & $0.029^{\mathrm{a}}$ & 1.40 & $0.48-4.09$ & 0.543 \\
\hline \multicolumn{7}{|l|}{ ECOG (o or 1 as reference) } \\
\hline ECOG 2 or more & 2.72 & $1.36-5 \cdot 53$ & $0.006^{\mathrm{a}}$ & 2.61 & $1.05-6.51$ & $0.040^{\mathrm{a}}$ \\
\hline PD-Lı any positivity (negative as reference) & 0.43 & $0.20-0.93$ & 0.427 & 0.31 & $0.10-0.90$ & $0.031^{\mathrm{a}}$ \\
\hline
\end{tabular}

PD-L1, programmed death-ligand 1; HR, hazard ratio; CI, confidence interval; EGFR, epidermal growth factor receptor; ECOG, Eastern Cooperative Oncology Group.

${ }^{\mathrm{a}} \mathrm{p}<0.05$ 
Supplementary Table 4. Multivariate analysis of overall survival after the administration of immune checkpoint inhibitor considering four different PD-L1 status

\begin{tabular}{|c|c|c|c|c|c|c|}
\hline \multirow{2}{*}{ Parameter } & \multicolumn{3}{|c|}{ Univariate analysis } & \multicolumn{3}{|c|}{ Multivariate analysis } \\
\hline & HR & $\mathrm{CI}$ & $p$ value & HR & $\mathrm{CI}$ & $p$ value \\
\hline Age & 0.99 & $0.96-1.03$ & 0.681 & 1.04 & $0.99-1.10$ & 0.117 \\
\hline Sex (female as reference) & 0.40 & $0.19-0.82$ & $0.013^{\mathrm{a}}$ & 4.22 & $0.37-47.6$ & 0.244 \\
\hline \multicolumn{7}{|l|}{ Smoking status (never smoker as reference) } \\
\hline Current/former smokers & 0.40 & $0.19-0.83$ & $0.014^{\mathrm{a}}$ & 0.07 & $0.01-0.79$ & $0.032^{\mathrm{a}}$ \\
\hline Histologic subtype (adenocarcinoma as reference) & & & 0.163 & & & 0.092 \\
\hline Squamous & 0.76 & $0.34-1.66$ & 0.484 & 1.73 & $0.54-5.50$ & 0.356 \\
\hline Others & 0.31 & $0.09-1.06$ & 0.061 & 0.30 & $0.08-1.11$ & 0.070 \\
\hline EGFR mutation (absent as reference) & 2.24 & $1.05-4.79$ & $0.038^{\mathrm{a}}$ & 2.42 & $0.78-7.47$ & 0.125 \\
\hline \multicolumn{7}{|l|}{ No. of prior chemotherapy regimen ( 1 or 2 as reference) } \\
\hline 3 or more & 2.22 & $1.09-4.53$ & $0.029^{\mathrm{a}}$ & 1.38 & $0.47-4.01$ & 0.555 \\
\hline \multicolumn{7}{|l|}{ ECOG (o or 1 as reference) } \\
\hline ECOG 2 or more & 2.72 & $1.36-5.53$ & $0.006^{\mathrm{a}}$ & 2.42 & $0.94-6.27$ & 0.068 \\
\hline PD-Lı classification (PD-L1 (-/-) as reference) & & & 0.160 & & & 0.179 \\
\hline $\mathrm{PD}-\mathrm{L1}(-/+)$ & 0.51 & $0.19-1.38$ & 0.185 & 0.30 & $0.09-1.04$ & 0.057 \\
\hline $\mathrm{PD}-\mathrm{L1}(+/-)$ & 0.28 & $0.08-1.02$ & 0.054 & 0.20 & $0.03-1.21$ & 0.080 \\
\hline $\mathrm{PD}-\mathrm{L} 1(+/+)$ & 0.45 & $0.19-1.07$ & 0.071 & 0.33 & $0.10-1.09$ & 0.069 \\
\hline
\end{tabular}

PD-L1, programmed death-ligand 1; HR, hazard ratio; CI, confidence interval; EGFR, epidermal growth factor receptor; ECOG, Eastern Cooperative Oncology Group.

${ }^{\mathrm{a}} \mathrm{p}<0.05$. 


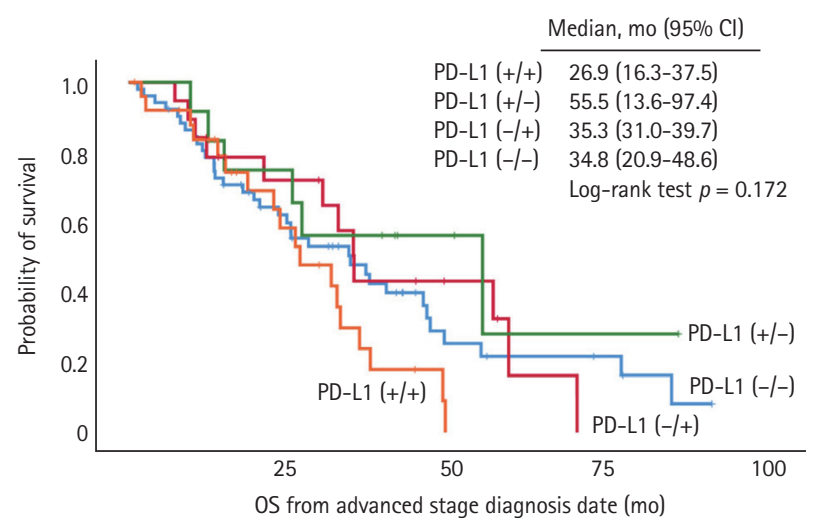

\section{No. at risk}

PD-L1 (+/+) 26

PD-L1 (+/-) 12

PD-L1 (-l+) 19

PD-L1 (-l-) 52

\section{A}

$\begin{array}{rlll}11 & 0 & 0 & 0 \\ 8 & 3 & 1 & 0 \\ 11 & 4 & 0 & 0 \\ 27 & 7 & 4 & 0\end{array}$

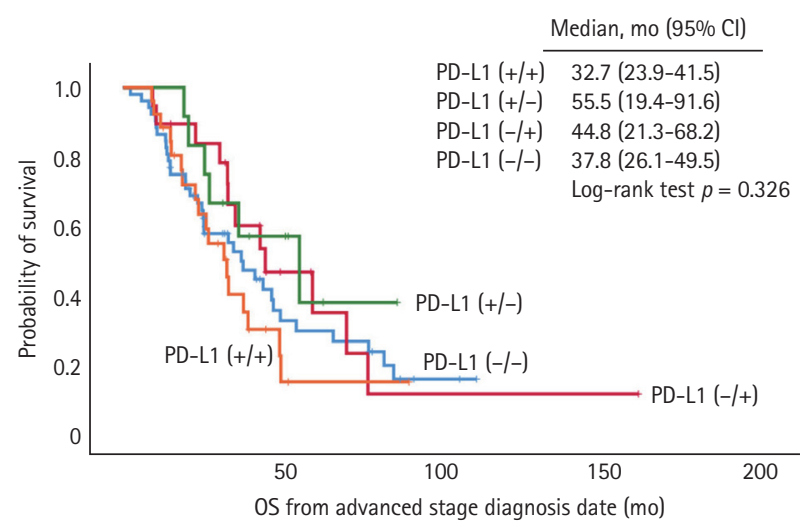

No. at risk

PD-L1 (+/+) 26

PD-L1 (+/-) 12

PD-L1 (-/+) 19

PD-L1 (-/-) 52

B

Supplementary Figure 1. Overall survival (OS) of patients in four groups categorized according to changes in programmed death-ligand 1 (PD-L1) expression. (A) There was no significant difference in the OS, calculated from the date of advanced diagnosis, among patients in the four groups $(p=0.172)$. (B) There was no significant difference in the OS, calculated from the date of initial diagnosis, among patients in the four groups $(p=0.326)$. CI, confidence interval. 

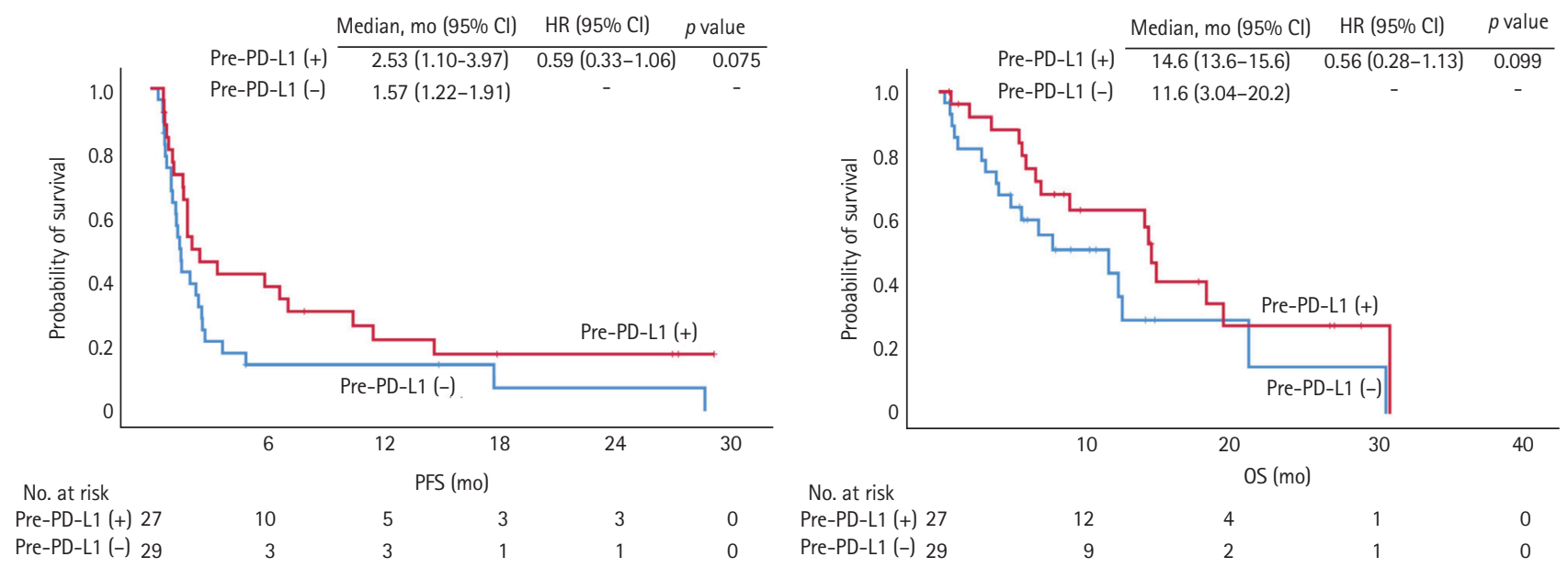

A

B

Supplementary Figure 2. Comparison of responses to immune checkpoint inhibitors between patients with programmed death-ligand 1 (PD-L1) positivity in archival samples (pre-PD-L1 [+] group) and those with PD-L1 negativity in archival samples (pre-PD-Li [-] group). There were no significant differences in (A) progression-free survival (PFS) and (B) overall survival (OS) between the two groups. HR, hazard ratio; CI, confidence interval. 

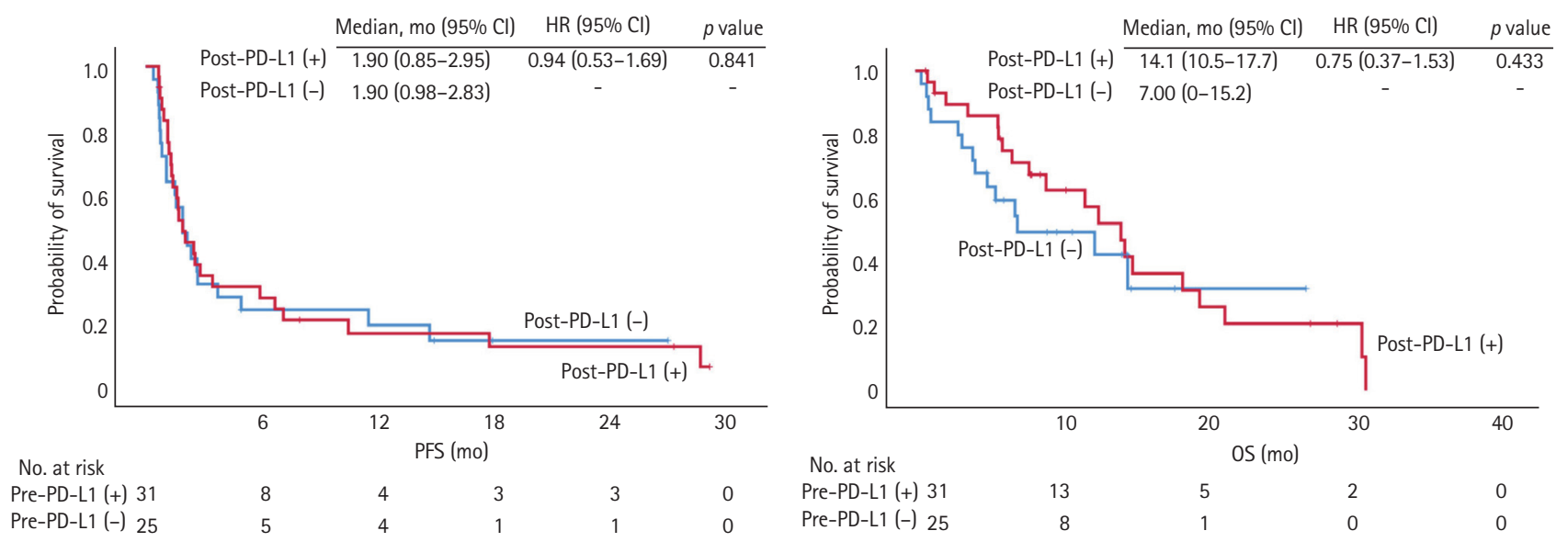

A

B

Supplementary Figure 3. Comparison of responses to immune checkpoint inhibitors between patients with programmed death-ligand 1 (PD-L1) positivity in recent samples (post-PD-L1 [+] group) and those with PD-L1 negativity in recent samples (post-PD-L1 [-] group). There were no significant differences in (A) progression-free survival (PFS) and (B) overall survival (OS) between the two groups. HR, hazard ratio; CI, confidence interval. 